Research Paper

\title{
Longitudinal FDG-PET Revealed Regional Functional Heterogeneity of Bone Marrow, Site-Dependent Response to Treatment and Correlation with Hematological Parameters
}

\author{
Masashi Yagi1,2, Jerry Froelich³, Luke Arentsen", Ryan Shanley5 , Rahel Ghebre1,6, Douglas Yee1,7, Susanta \\ $\mathrm{Hui}^{1,4}{ }^{凶}$ \\ 1. Masonic Cancer Center, University of Minnesota, Minneapolis, MN, USA \\ 2. Osaka University Graduate School of Medicine, Suita, Osaka, Japan \\ 3. Department of Radiology, University of Minnesota, Minneapolis, MN, USA \\ 4. Department of Therapeutic Radiology, Medical School, University of Minnesota, Minneapolis, MN, USA \\ 5. Biostatistics Core, Masonic Cancer Center, University of Minnesota, Minneapolis, MN, USA \\ 6. Department of Obstetrics and Gynecology, University of Minnesota, Minneapolis, MN, USA \\ 7. Department of Medicine, Medical School, University of Minnesota, Minneapolis, MN, USA
}

$\triangle$ Corresponding author: Susanta K Hui, PhD, DABR, Associate Professor, Masonic Cancer Center, University of Minnesota, Minneapolis, MN, USA, Email: huixx019@umn.edu, TEL: 612-626-4484, FAX: 612-626-7060

() 2015 Ivyspring International Publisher. Reproduction is permitted for personal, noncommercial use, provided that the article is in whole, unmodified, and properly cited. See http://ivyspring.com/terms for terms and conditions.

Received: 2014.12.14; Accepted: 2015.02.10; Published: 2015.04.15

\begin{abstract}
Purpose: The purposes of this study were: 1) to show bone marrow (BM) functional heterogeneity, 2) to demonstrate site-dependent responses of BM to cancer treatment utilizing whole body FDG-PET/CT and 3) to identify correlations between FDG uptake in different bone sites and long term complete blood count (CBC).

Methods: Thirty two patients who had pre- and post-treatment FDG-PET/CT scans were selected retrospectively. Each patient received either head and neck radiation for cancer of the tongue, or pelvic radiation for rectal or cervical cancer with chemotherapy. Patients had FDG-PET/CT performed prior to the first radiation therapy session and at least one FDG-PET/CT after completion of the prescribed radiation therapy.

Results: FDG uptake before radiotherapy was significantly different among bone regions $(p<$ 0.01 ). This heterogeneity was felt to reflect site-dependent amounts of BM contents possibly due to structural and functional requirements. FDG uptake in the irradiated regions was significantly decreased on the first and second follow-ups after radiation. Feasibly, this could be due to a reduction in the number of active BM cells following intensive radiation in addition to concurrent chemotherapy. Overall, $\mathrm{CBC}$ significantly decreased after treatment. Correlation values of each hematological parameter with FDG uptake varied among skeletal regions and scan time points. FDG uptake in sacrum and lumbar regions had better correlation with white blood cells and neutrophils.

Conclusions: Longitudinal FDG-PET revealed a regional functional heterogeneity of the BM site-dependent response to treatment. Patients experienced immediate and prolonged marrow metabolic damage that correlates with hematological parameters. FDG-PET/CT may provide additional capabilities to assess BM health.
\end{abstract}

Key words: FDG-PET/CT, Bone marrow, CBC, metabolic heterogeneity 


\section{Introduction}

Bone marrow (BM), a key component of the hematopoietic and lymphatic system, is anatomically and physiologically distributed through the whole body and produces blood cells that support the body's biological system. Multiple insults from both the cancer and treatments including radiation and chemotherapy are responsible for BM damage. A bone marrow aspiration and biopsy remains the gold standard to evaluate BM and is mandatory to reach a specific diagnosis. A biopsy, however, intrinsically assumes a homogeneous BM distribution and function. The procedure is invasive and limited by sampling error [1].

Whole body ${ }^{18} \mathrm{~F}$ labeled fluoro-2-deoxy-2-D-glucose (FDG) computed tomography (CT) guided positron emission tomography (PET) is one of the novel non-invasive methods for assessment of BM. FDG-PET can provide information about functioning BM [2] and its anatomic and physiologic distribution as well as detection of pathologic lesions as demonstrated by increased glucose metabolism [3]. FDG accumulates physiologically in BM as glycolysis occurs [4]. The pathophysiological significance of changes in FDG activity in the BM is still unclear. Understanding of the physiology of FDG uptake into BM might facilitate identification of regional heterogeneity and the impact of combined treatment (e.g. radiotherapy and chemotherapy) on BM metabolism.

Neutropenia is a severe dose-limiting side effect of myelosuppressive chemotherapy in patients with solid tumors [5-7]. As a result of the hematopoietic system's sensitivity to the treatment, monitoring complete blood counts (CBC) has become routine for most patient during chemotherapy treatment. Monitoring $\mathrm{CBC}$ is one of the standard tests to examine chemotherapy toxicity. There has been documentation of a strong correlation between FDG uptake in thoracic and/or lumbar spine and white blood cell counts (WBCs) in patients before undertaking treatment [8]. Elicin et al. observed early effects of treatment on BM metabolism using FDG-PET and its association with WBCs, one of many hematological parameters [9]. However, it is unclear whether metabolic function is region dependent or if the metabolic function at different skeletal regions also correlates with different hematologic parameters. It is also unknown whether cancer associated treatment induces prolonged damage to BM and whether FDG uptake correlates with hematological parameters for longer term follow-up. This information could support the utility of FDG-PET imaging for diagnosing BM involvement by malignancy, thus altering the strategy for targeted treatment and assist in the evaluation of BM damage caused by therapies.
The purposes of this study were: 1) to show BM functional heterogeneity, 2) to demonstrate a site-dependent response of BM to treatment utilizing whole body FDG-PET/CT in two different groups (head and neck cancer, and cervical and rectum cancer) who frequently undergo FDG-PET/CT scan for tumor response, and 3) to identify correlation between FDG uptake in different skeletal sites and CBC over a longer term.

\section{Methods and Materials}

\section{Patients inclusion}

Thirty-two patients who had pre- and post-treatment FDG-PET/CT scans between 2006 and 2011 were selected retrospectively from the University of Minnesota hospital system. Eligible patients had no previous chemotherapy or radiotherapy. Each patient received either head \& neck radiation for cancer of the tongue (hereafter $H \& N, n=14$ ), or pelvic radiation for rectal or cervical cancer (hereafter Pelvis, $n=18$ ) with chemotherapy. Three-dimensional conformal radiotherapy or intensity modulated radiotherapy (IMRT) were used for H\&N cancer. Chemotherapy for H\&N cancer patients was concomitant to the radiotherapy. Patients must have had an FDG-PET/CT performed prior to the first radiation therapy session and had at least one FDG-PET/CT since completion of the prescribed radiation therapy. The time period between the baseline PET/CT scan and the first and second follow-up were $5.3 \pm 2.6$ and $14.1 \pm 4.0$ months, respectively. Patients were anonymized after acquisition of PET/CT images and pertinent demographic data was removed. The average age was $56.8 \pm 13.5$ years old. Each patient's chart was thoroughly analyzed for a record of any metastatic disease. Metastases were recorded even if they did not involve bone. This study was approved by the University of Minnesota Institutional Review Board.

\section{Blood glucose measurements}

Blood glucose measurement was performed just before the FDG injection. The mean blood glucose value of the thirty-two patients was $107.0 \pm 17.6 \mathrm{mg} / \mathrm{dl}$, and ranged from $74 \mathrm{mg} / \mathrm{dl}$ to $165 \mathrm{mg} / \mathrm{dl}$.

\section{PET/CT imaging}

All FDG-PET/CT studies were performed 60-90 min after intravenous administration of $558 \pm 48 \mathrm{MBq}$ of FDG and after fasting for at least 4 hours. The scanner used for all subjects was a Siemens Biograph-16 fitted with high resolution crystals (HD-PET). Scanner was cross calibrated between the dose calibrator and scanner and I clocks were synchronized. The PET-CT protocol started with CT images acquired from the head to upper thigh. A 
whole-body emission PET scan of the same region followed at $3 \mathrm{~min}$ per table position at a resolution of $220 \times 220$. The PET and CT images were co-registered using the positional information from the table and patient. Attenuation corrections of PET images were performed using the CT data. PET images were reconstructed with an iterative reconstruction with ordered-subset expectation maximization algorithm.

\section{Image analysis}

Three-dimensional PET/CT fusion images were used to manually set three-dimensional regions of interest (ROI) on the BM in bilateral glenoid fossa, bilateral proximal humera, first cervical vertebra, first thoracic vertebra, first lumbar vertebra, first sacral vertebra, bilateral femoral heads, bilateral femoral necks, and bilateral proximal femurs. The ROI were measured to an area of $65 \pm 3 \mathrm{~cm}^{3}$ for all points of interest. Standardized uptake value (SUV) was used to evaluate FDG uptake by BM. SUV was calculated as follows: $\mathrm{SUV}=$ [measured activity concentration $(\mathrm{Bq} / \mathrm{ml})$ in ROI] / [injected activity $(\mathrm{MBq}) /$ body weight $(\mathrm{kg})$ ]. Heterogeneity is described as a varying uptake distribution of tracer among skeletal sites in this study.

\section{Hematologic Parameters}

The hematological data, including WBC count $\left(\times 10^{3} / \mu \mathrm{l}\right)$, red blood cell $(\mathrm{RBC})$ count $\left(\times 10^{6} / \mu \mathrm{l}\right)$, hemoglobin concentration $(\mathrm{Hb} ; \mathrm{g} / \mathrm{dl})$, hematocrit $(\mathrm{Ht}$; $\%)$ and platelet count $\left(\times 10^{4} / \mu \mathrm{l}\right)$, were recorded from laboratory studies performed before or after the PET/CT examinations. In 32 patients, neutrophil, lymphocyte, monocyte, eosinophil and basophil counts were also available, but the monocyte, eosinophil and basophil counts were excluded from the analysis because of their low values. The median interval between the hematological examination and the PET study was 7 days.

\section{Statistical analysis}

Change in FDG uptake among all skeletal re-
Two-sided-paired t-tests assessed differences in FDG uptake and hematological parameters between baseline and each follow-up. Correlations between FDG uptake and hematological parameters were evaluated by Pearson's correlation coefficient. All statistical analyses were performed with Microsoft Excel 2010 (Microsoft, Redmond, WA, USA).

\section{Results}

\section{Bone marrow functional heterogeneity}

Heterogeneity is described as varying uptake distribution of a tracer among skeletal sites in 32 patients in this study. FDG uptake before radiotherapy was significantly different among bone regions (Fig. 1, $p<0.01)$. Vertebrae had higher activity than the other regions. FDG uptake also differed within a bone site (i.e. vertebrae and femur). Lumber vertebra had the highest uptake in the vertebral column. Femoral neck had the highest uptake in the femur.

\section{Treatment response of bone marrow}

Figures 2A and 3A show schematic representations of radiation fields for $\mathrm{H} \& \mathrm{~N}$ and Pelvis treatments, respectively. FDG uptake in the irradiated regions was significantly decreased on the first and second follow-up after radiation (Figs. 2B and 3B, $p<$ $0.05)$. Average FDG uptake change within an irradiated region between baseline and the first follow-up, and baseline and the second follow-up were $21.7 \%$ and $31.6 \%$ for H\&N cases, and $28.6 \%$ and $31.9 \%$ for Pelvis cases, respectively. The change patterns were different between these groups. The degree of the decrease was similar in patients treated for H\&N cancer between baseline to the first follow-up, and the first to the second follow-up (Fig. 2C). A smaller change was observed in the first to the second follow-up than baseline to the first follow-up in the Pelvis treatment group (Fig. 3C). The uptake was not recovered by the second follow-up in both groups (Figs. 2B, C and Figs. 3B, C, $p<0.05$ ). gions was analyzed by single factor ANOVA.

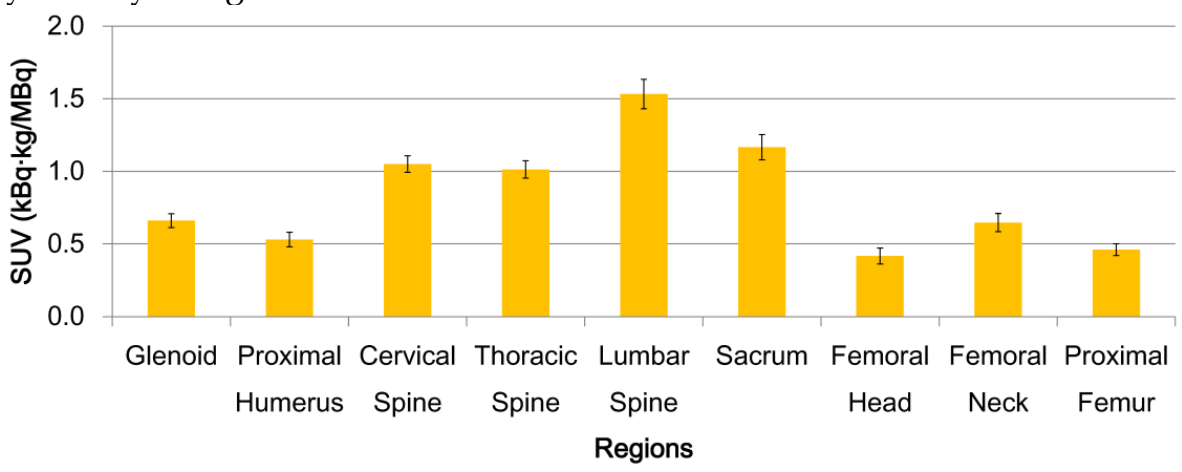

Figure 1. BM functional heterogeneity for all patients. Mean FDG uptake and standard error bars are shown. FDG uptake is significantly different among bone regions $(p<0.01)$. Lumber vertebra has the highest uptake in the spinal column. Femoral neck has the highest uptake in the femur. 

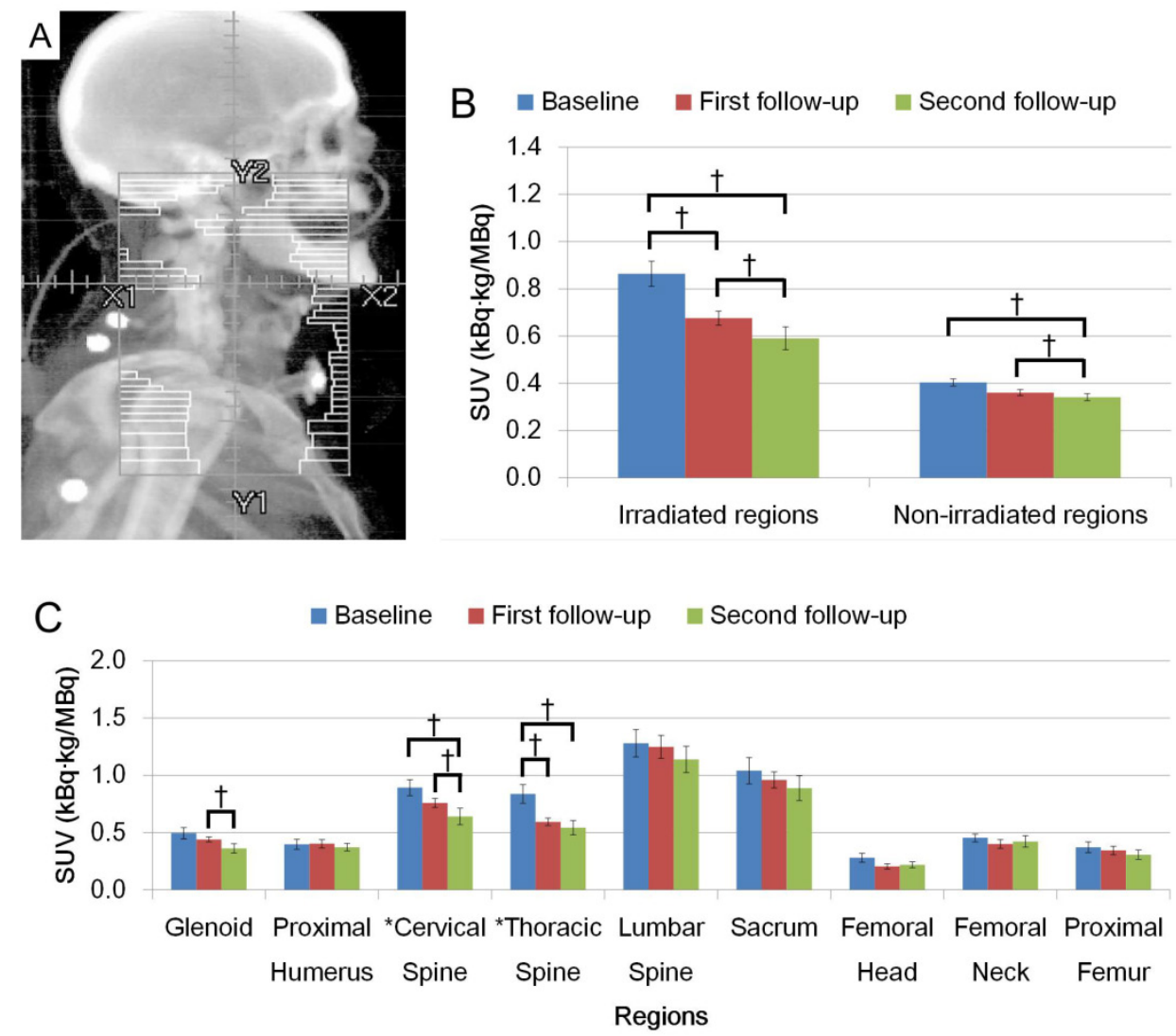

Figure 2. (A) Representation of radiation fields for H\&N irradiation. (B) FDG uptake in irradiated region and non-irradiated region. (C) FDG uptake among different anatomical regions. $(\dagger: p<0.05)$.

When uptake changed within an irradiated region, the extent of the change was dramatically different among the anatomical locations. Uptake change in cervical vertebra showed larger decrease in the second follow-up from the first follow-up while the thoracic vertebra had an opposite pattern in $H \& N$ cases. In Pelvis cases, the change in uptake at the sacrum was the largest in each observed period with a larger change in the first follow-up. A degree of uptake change within femur showed that the largest change was observed in femoral neck.

Non-irradiated regions in H\&N cases showed significant decreases in FDG uptake (Fig. 2B). Several bones outside the radiation field showed dramatic change after radiation; specifically the glenoids in the second follow-up in the H\&N group (Fig. 2C, $p<0.05$ ) and cervical vertebra in the Pelvis group (Fig. 3 C, $p<$ 0.05).

\section{Correlation with CBC}

Table 1 shows time course change of hematological parameters from 32 patients. Overall, CBC significantly decreased after treatment. CBC parameters, excluding hemoglobin, were decreased up to the second follow-up. Lymphocytes showed the greatest decline after treatment.
Table 1. Time course change of hematological parameters.

\begin{tabular}{lcccccc}
\hline & \multicolumn{2}{c}{$\begin{array}{c}\text { Baseline } \\
(\mathbf{n}=\mathbf{2 9})\end{array}$} & \multicolumn{2}{c}{$\begin{array}{c}\text { First follow-up } \\
(\mathbf{n = 2 9 )}\end{array}$} & \multicolumn{2}{c}{$\begin{array}{c}\text { Second follow-up } \\
(\mathbf{n = 1 4 )}\end{array}$} \\
\hline Blood cell type & Mean & SD & Mean & SD & Mean & SD \\
\hline $\mathrm{WBC}^{*} \dagger$ & 9.4 & $(5.2)$ & 6.4 & $(6.0)$ & 5.7 & $(1.4)$ \\
$\mathrm{RBC}^{*} \dagger$ & 4.4 & $(0.6)$ & 4.0 & $(0.5)$ & 3.9 & $(0.6)$ \\
Hemoglobin $^{*} \dagger$ & 12.9 & $(1.8)$ & 12.0 & $(1.6)$ & 12.3 & $(1.6)$ \\
Hematocrit $^{*} \dagger$ & 38.6 & $(4.5)$ & 35.8 & $(4.2)$ & 36.4 & $(4.0)$ \\
Platelet $^{*}$ & 269.6 & $(72.5)$ & 227.5 & $(53.5)$ & 221.0 & $(60.8)$ \\
Neutrophil $^{*} \dagger$ & 6.8 & $(4.7)$ & 5.2 & $(6.2)$ & 4.0 & $(1.8)$ \\
Lymphocyte* $^{*} \dagger$ & 1.8 & $(1.3)$ & 0.7 & $(0.3)$ & 0.8 & $(0.5)$ \\
Monocyte $^{*} \dagger$ & 0.7 & $(0.3)$ & 0.5 & $(0.2)$ & 0.4 & $(0.2)$ \\
\hline
\end{tabular}

Overall CBC significantly decreased after treatment. CBC excepting for hemoglobin kept significant low level on the second follow-up. Lymphocyte showed the greatest decline after treatment.

* Baseline of CBC (WBC, RBC, hemoglobin, Hematocrit, Platelet, Neutrophil,

Lymphocyte, and Monocyte) significantly decreased compared with first follow-up $(\mathrm{p}<0.05)$.

$\dagger$ Baseline of CBC (WBC, RBC, hemoglobin, Hematocrit, Neutrophil, Lymphocyte, and Monocyte) significantly decreased compared second follow-up $(\mathrm{p}<0.05)$.

Table 2 shows the correlation between regional FDG uptake and WBC or neutrophil. Correlation values of each parameter with FDG uptake varied among skeletal regions and scan time points. The sacrum and lumbar had the first and second highest correlation with WBC on baseline and the first follow-up, respectively. On the second follow-up, the lumbar vertebra had the highest correlation. 
Table 2. Correlation between regional FDG uptake and WBC or neutrophil

\begin{tabular}{|c|c|c|c|c|c|c|}
\hline \multirow[b]{2}{*}{ Regions } & \multicolumn{3}{|c|}{ WBC } & \multicolumn{3}{|c|}{ Neutrophil } \\
\hline & Baseline & $\begin{array}{c}\text { First } \\
\text { follow-up }\end{array}$ & $\begin{array}{c}\text { Second } \\
\text { follow-up }\end{array}$ & Baseline & $\begin{array}{c}\text { First } \\
\text { follow-up }\end{array}$ & $\begin{array}{l}\text { Second } \\
\text { follow-up }\end{array}$ \\
\hline Glenoid & 0.24 & -0.06 & -0.07 & 0.19 & -0.06 & 0.08 \\
\hline $\begin{array}{l}\text { Proximal } \\
\text { Humerus }\end{array}$ & 0.21 & -0.22 & -0.04 & 0.20 & -0.20 & 0.09 \\
\hline $\begin{array}{l}\text { Cervical } \\
\text { Spine }\end{array}$ & 0.05 & -0.19 & -0.26 & 0.00 & -0.19 & -0.25 \\
\hline $\begin{array}{l}\text { Thoracic } \\
\text { Spine }\end{array}$ & 0.24 & -0.05 & -0.11 & 0.16 & -0.02 & 0.04 \\
\hline $\begin{array}{l}\text { Lumbar } \\
\text { spine }\end{array}$ & $0.37^{*}$ & 0.23 & 0.37 & 0.33 & 0.24 & 0.40 \\
\hline Sacrum & $0.39^{*}$ & 0.32 & 0.28 & $0.40^{*}$ & 0.32 & 0.36 \\
\hline $\begin{array}{l}\text { Femoral } \\
\text { Head }\end{array}$ & 0.01 & 0.01 & -0.29 & 0.02 & 0.02 & -0.17 \\
\hline $\begin{array}{l}\text { Femoral } \\
\text { Neck }\end{array}$ & 0.18 & -0.04 & 0.17 & 0.15 & -0.04 & 0.34 \\
\hline $\begin{array}{l}\text { Proximal } \\
\text { Femur }\end{array}$ & 0.09 & 0.19 & 0.00 & 0.08 & 0.19 & 0.14 \\
\hline
\end{tabular}

Correlation values of each parameter with FDG uptake varied among skeletal regions and scan time points. Sacrum and lumbar were the first and second highest correlation between baseline and the first follow-up, respectively. On the second follow-up, the lumbar spine had the highest correlation.

* Correlation is significantly different from $0(\mathrm{p}<0.05)$.

\section{Discussion}

Longitudinal whole body FDG-PET imaging revealed regional heterogeneity in BM (i.e. BM metabo- lism differences) and site dependent responses to radiation by showing a varying amount of the tracer uptake. This study was the first attempt to explain BM functional heterogeneity and the response to cancer treatment within multiple skeletal sites as demonstrated by FDG-PET/CT images and quantitation. By comparing FDG uptake with hematologic parameters, we are the first to show a strong correlation between FDG uptake and values on long term post treatment follow up.

Our study showed variability of FDG uptake depending on the bone sites. FDG uptake can define active $\mathrm{BM}$ regions [2] and correlates with the number of hematopoietic marrow cells [10]. This heterogeneity could reflect site-dependent amount of BM contents possibly due to structural and functional differences [11, 12]. FDG uptake variations within individual bones were also observed, demonstrating BM heterogeneous distribution even in a single bone site. This heterogeneous distribution within the bone may be caused by of anatomical vascular supply differences. For example, in the femoral neck there is an optimal supply of blood via nutrient arteries from the femoral circumflex artery penetrating the femoral neck region, in contrast to the femoral head and
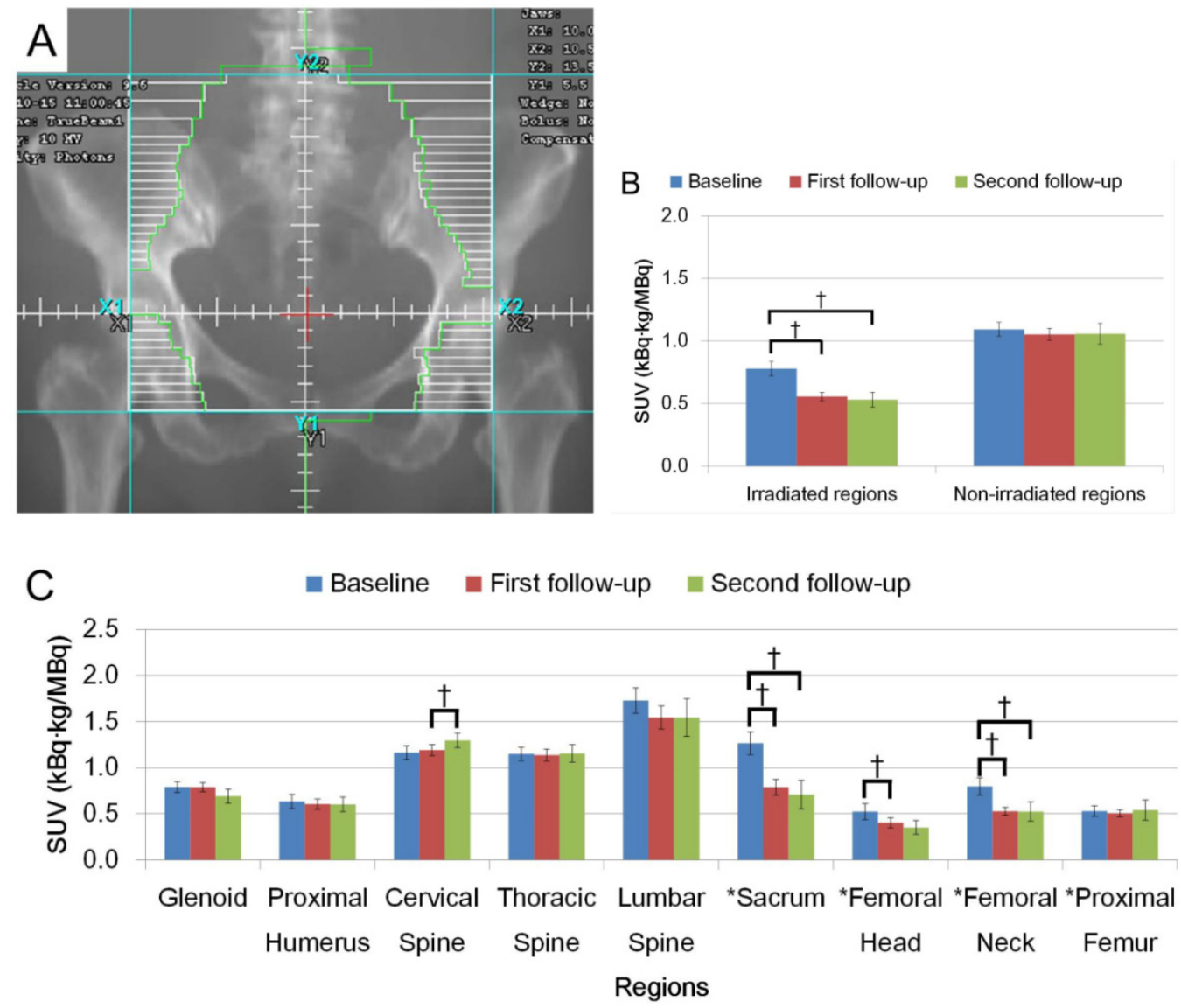

Figure 3. (A) Representation of radiation fields for Pelvic irradiation. (B) FDG uptake in irradiated region and non-irradiated region. (C) FDG uptake among different anatomical regions. FDG uptake is shown with SUV. $(\dagger: p<0.05)$. 
proximal femur [13]. Understanding regional functional differences in the BM may be crucial for: a) mapping sections of the BM to identify the regions that are most affected by disease or clinical intervention rather than random selection of $\mathrm{BM}$ regions to assay, b) beginning to understand the role that local and whole body BM environments may play in the interaction with systemic endocrine and immune elements, and c) evaluating the response to disease management that incorporate factors such skeletal site and BM composition.

FDG uptake within the radiation field was dramatically decreased after treatment. This may be due to a reduction in the number of active BM cells following intensive radiation in addition to concurrent chemotherapy $[2,10]$. The degree of the reduction varied across irradiated bone sites and scan time points, possibly sustaining site-dependent response to radiation. The amount of $\mathrm{BM}$ at a skeletal site and/or its distribution within the site at baseline could correlate with the variation of FDG uptake after treatment. FDG-PET has been shown to identify hematologically active BM regions in which radiation dose is associated with hematologic toxicity [2] and leukemia risk [14]. Since we have demonstrated a site-dependent response to radiation, radiation therapy treatment plan could be further optimized and/or adapted to improve the radiation delivery by utilizing such techniques as IMRT or dose painting. This may, in turn, reduce toxicity and prevent delays in treatment delivery. Whole body FDG-PET/CT could be a tool to develop a comprehensive understanding of radiation-induced effects on BM metabolism by investigating the temporal (short- and long-term) and spatial (local vs distant) effects of radiation on bone structure and function.

Non-irradiated bone sites showed significant decrease of FDG uptake. This reduction could happen due to combinations of chemotherapy, scattered radiation dose and abscopal effect. A dominant effect contributing to the decrease would depend on a location of a bone relative to a radiation field. In this study, FDG uptake in the glenoids, for example, was significantly decreased in H\&N cases. The bone site was close to the radiation field as shown in figure $2 \mathrm{~A}$ and the reduction could be mainly explained with a scattered radiation dose.

FDG uptake in sacrum and lumbar had better correlation with WBC and neutrophil. The good correlation with neutrophil is due to a) that the neutrophil cell series are predominant in BM, b) the main energy production pathway in neutrophil is glycolysis and c) the short life span (about $10 \mathrm{~h}$ ), reflecting granulopoiesis at the time blood was collected. Since the counts of other blood cells are proportionally less and have a longer life span, there was a lower correlation with FDG uptake. Since both of the sites are BM rich regions, these sites would reflect the hematopoietic activity more predominantly than other BM regions. This result could indirectly support the site-dependent BM function. FDG uptake after the second follow-up still showed a lower uptake level than baseline, reflecting persistence of BM damage as the CBC declined. Correlation of FDG uptake in sacrum with the WBC parameters could therefore dramatically decrease on the second follow-up. This continuing damage would be the number of BM cell reduced due to a replacement of BM with marrow adipose tissue after treatment. An increase in marrow adiposity could potentially lead to an increase in bone fracture due to loss of bone density. Clinically, FDG-PET/CT represents diffusely increased FDG uptake by BM or transient increases of uptake, termed "flares" [15]. It is crucial to differentiate these cases from increased uptake due to other causes on PET images. Current knowledge on $\mathrm{CBC}$ could support the interpretation of FDG-PET images for diagnosing $\mathrm{BM}$ involvement by malignancy. This provides a strategy for attacking the disease and assessing BM recovering from treatment damage.

Translational opportunity: Patients experiencing marrow damage are often unable to receive their subsequent dose of chemotherapy on time or in full, thus yielding a negative impact on the success of the cancer treatment $[6,7]$. Delivery of the planned chemotherapy dose is particularly important where the treatment goal is for cure or extension of life. Patients who experience significant treatment-related CBC side effects may benefit from greater support to maintain bone health. Furthermore, CBC changes while receiving radiation or chemotherapy can potentially indicate greater long term treatment damage.

This study had several limitations. This study was a retrospective analysis and needs a prospective cohort to affirm these findings. Only patients who were diagnosed with a malignancy of $\mathrm{H} \& \mathrm{~N}$, cervix or rectum were recruited for this study, thus it is uncertain whether our findings are specific to these diseases. The time of the first PET/CT scans and second PET/CT scans were not controlled and cross-sectional comparisons should be done with caution. Radiation dose and extension of the field varied. Similarly, there are variations in chemotherapy dose and the duration of treatment. In this study, the goal was to measure FDG uptake changes in pre- and post-treatment for the same patient population. Sample size was limited. Healthy controls may help to identify the relative risk of bone loss in different treatment groups and healthy subjects. 


\section{Conclusion}

Longitudinal FDG-PET/CT revealed regional functional heterogeneity of BM, site-dependent response to treatment and correlation with hematological parameters. BM function differs not only throughout the skeleton but also within an individual bone site. The response of $\mathrm{BM}$ to a treatment depends on the bone site. CBC such as WBC and neutrophil has a correlation to FDG uptake in BM-rich skeletal regions. A prospective longitudinal study conjunction with molecular and biochemical mechanisms will be further conducted.

\section{Acknowledgement}

This work was supported by the National Institute of Health grants (1R01CA154491 and P30 CA77598), the National Center for Advancing Translational Sciences of the National Institutes of Health Award Number UL1TR000114, Grant in Aid from the University of Minnesota, Rosenthal fund, and Japan Society for the Promotion of Science Core to Core Program (23003).

\section{Competing Interests}

The authors have declared that no competing interest exists.

\section{References}

1. BRUNNING RD, BLOOMFIELD CD, McKENNA RW, PETERSON L. Bilateral trephine bone marrow biopsies in lymphoma and other neoplastic diseases. Annals of internal medicine. 1975; 82: 365-6.

2. Rose BS, Liang Y, Lau SK, Jensen LG, Yashar CM, Hoh CK, et al. Correlation Between Radiation Dose to $<$ sup $>18<$ /sup $>$ F-FDG-PET Defined Active Bone Marrow Subregions and Acute Hematologic Toxicity in Cervical Cancer Patients Treated With Chemoradiotherapy. International Journal of Radiation Oncology* Biology* Physics. 2012; 83: 1185-91.

3. Yagi M, Arentsen L, Shanley RM, Rosen CJ, Kidder LS, Sharkey LC, et al. A Dual-Radioisotope Hybrid Whole-Body Micro-Positron Emission Tomography/Computed Tomography System Reveals Functional Heterogeneity and Early Local and Systemic Changes Following Targeted Radiation to the Murine Caudal Skeleton. Calcified tissue international. 2014;: $1-9$.

4. Shyh-Chang N, Daley GQ, Cantley LC. Stem cell metabolism in tissue development and aging. Development. 2013; 140: 2535-47.

5. Barni S, Lorusso V, Giordano M, Sogno G, Gamucci T, Santoro A, et al. A prospective observational study to evaluate G-CSF usage in patients with solid tumors receiving myelosuppressive chemotherapy in Italian clinical oncology practice. Medical oncology (Northwood, London, England). 2014; 31: 797. doi:10.1007/s12032-013-0797-z.

6. Chirivella I, Bermejo B, Insa A, Pérez-Fidalgo A, Magro A, Rosello S, et al. Optimal delivery of anthracycline-based chemotherapy in the adjuvant setting improves outcome of breast cancer patients. Breast cancer research and treatment. 2009; 114: 479-84.

7. Pettengell R, Schwenkglenks M, Leonard R, Bosly A, Paridaens R, Constenla $\mathrm{M}$, et al. Neutropenia occurrence and predictors of reduced chemotherapy delivery: results from the INC-EU prospective observational European neutropenia study. Supportive care in cancer. 2008; 16: 1299-309.

8. Murata Y, Kubota K, Yukihiro M, Ito K, Watanabe H, Shibuya H. Correlations between < sup > 18</sup> F-FDG uptake by bone marrow and hematological parameters: measurements by PET/CT. Nuclear medicine and biology. 2006; 33: 999-1004.

9. Elicin O, Callaway S, Prior JO, Bourhis J, Ozsahin M, Herrera FG. [< sup> $18</$ sup $>$ F] FDG-PET Standard Uptake Value as a Metabolic Predictor of Bone Marrow Response to Radiation: Impact on Acute and Late Hematological Toxicity in Cervical Cancer Patients Treated With Chemoradiation Therapy. International Journal of Radiation Oncology* Biology* Physics. 2014
10. Chen C, Yan L-M, Guo K-Y, Wang Y-J, Zou F, Gu W-W, et al. The diagnostic value of $[18 \mathrm{~F}]-\mathrm{FDG}-\mathrm{PET} / \mathrm{CT}$ in hematopoietic radiation toxicity: a Tibet minipig model. Journal of radiation research. 2012; 53: 537-44.

11. Ritman EL, Bolander ME, Fitzpatrick LA, Turner RT. Micro-CT imaging of structure-to-function relationship of bone microstructure and associated vascular involvement. Technology and Health Care. 1998; 6: 403-12.

12. Turner $\mathrm{CH}$, Hsieh YF, Müller R, Bouxsein ML, Rosen CJ, McCrann ME, et al. Variation in bone biomechanical properties, microstructure, and density in $\mathrm{BXH}$ recombinant inbred mice. Journal of Bone and Mineral Research. 2001; 16: 206-13.

13. Tuljapurkar SR, McGuire TR, Brusnahan SK, Jackson JD, Garvin KL, Kessinger $\mathrm{MA}$, et al. Changes in human bone marrow fat content associated with changes in hematopoietic stem cell numbers and cytokine levels with aging. Journal of anatomy. 2011; 219: 574-81.

14. Zhang Y, Yan Y, Nath R, Bao S, Deng J. Personalized estimation of dose to red bone marrow and the associated leukaemia risk attributable to pelvic kilo-voltage cone beam computed tomography scans in image-guided radiotherapy. Physics in medicine and biology. 2012; 57: 4599.

15. Trigonis I, Jackson A. Imaging pharmacodynamics in oncology: the potential significance of "flares". Annals of nuclear medicine. 2010; 24: 137-47. 